\title{
Efeitos da invasão biológica de algaroba - Prosopis juliflora (Sw.) DC. sobre a composição e a estrutura do estrato arbustivo-arbóreo da caatinga no Município de Monteiro, PB, Brasil
}

\author{
Cláudia Maria Alves Pegado ${ }^{1,5}$, Leonaldo Alves de Andrade $^{2}$, Leonardo Pessoa Félix ${ }^{3}$ e Israel Marinho Pereira
}

Recebido em 31/10/2005. Aceito em 18/05/2006

\begin{abstract}
RESUMO - (Efeitos da invasão biológica de algaroba - Prosopis juliflora (Sw.) DC. sobre a composição e a estrutura do estrato arbustivo-arbóreo da caatinga no Município de Monteiro, PB, Brasil). A contaminação biológica é uma das principais causas da perda de biodiversidade atualmente no planeta. Este trabalho teve como objetivo avaliar os impactos causados pela invasão biológica de Prosopis juliflora sobre a composição e a estrutura do componente arbustivo-arbóreo da caatinga, tanto no estrato adulto quanto na regeneração natural. Foram plotadas 20 parcelas de $400 \mathrm{~m}^{2}$, sendo $10 \mathrm{em}$ um remanescente de caatinga bem conservada (Ambiente I) e 10 em uma área de caatinga invadida por $P$. juliflora (Ambiente II). A área invadida apresentou-se muito mais pobre em relação à primeira, em todos os parâmetros avaliados. Os impactos causados pela invasora foram sentidos tanto na estrutura, quanto na diversidade biológica da comunidade. A Densidade Relativa de P. juliflora no estrato adulto da área invadida atingiu cerca de $90 \%$, o que reflete a sua capacidade de competição e eliminação das demais espécies. O índice de diversidade de Shannon-Weaver (H') apresentou os valores de 2,81 e 0,61 para o componente adulto dos Ambientes I e II, respectivamente. Para a regeneração natural os valores desse índice foram de 2,31 e 1,14 para os mesmos Ambientes. Conclui-se que P. juliflora forma densos maciços populacionais e compete com as espécies nativas, afetando severamente a composição florística, a diversidade e a estrutura das comunidades autóctones invadidas.
\end{abstract}

Palavras-chave: contaminação biológica, Prosopis juliflora, perda de biodiversidade, caatinga

\begin{abstract}
Effects of the biological invasion of algaroba - Prosopis juliflora (Sw.) DC. on composition and structure of the shrubtree stratum of the caatinga in Monteiro Municipality, Paraíba State, Brazil). Biological contamination is currently one of the main causes of biodiversity loss on the planet. The aim of this study was to evaluate the impacts caused by $P$. juliflora invasion on caatinga phytodiversity in Monteiro Municipality, in both the adult stratum and natural regeneration. Twenty plots of $400 \mathrm{~m}^{2}$ were set up, 10 in a conserved caatinga fragment (Environment I) and 10 in a caatinga area invaded by $P$. juliflora (Environment II). The invaded area was much poorer in relation to the conserved area for all parameters investigated. The impacts caused by the alien species were felt in structure as well in community biological diversity. The relative density of $P$. juliflora in the adult stratum of the invaded area was ca. $90 \%$, which reflects its capacity to compete and eliminate other species. The Shannon-Weaver diversity index (H') had values of 2.81 and 0.61 for the adult component of Environments I and II, respectively. For natural regeneration, this index was 2.31 and 1.14 for the same sites. It was concluded that $P$. juliflora forms dense populations that grow along with native species, severely affecting the floristic composition, diversity and structure of autochthonous communities
\end{abstract}

Key words: biological contamination, Prosopis juliflora, biodiversity loss, caatinga

\section{Introdução}

Grande parte do Estado da Paraíba encontra-se sob o domínio das caatingas. Estas são regiões marcadas pela concentração das precipitações pluviométricas em um curto período de tempo, quase sempre inferior a três meses do ano, estando os demais meses sob condição de elevado déficit hídrico e forte insolação (Andrade et al. 1999).
Historicamente as secas têm sido apontadas como a causa dos grandes problemas do semi-árido, embora se saiba que estas apenas agravam as seqüelas ecos-sociais da região. $\mathrm{Na}$ busca de soluções para estes problemas, tem-se recorrido às mais diversas estratégias a maioria delas, porém, pautaram-se na importação de modelos, tecnologias e ou insumos que, posteriormente, se mostraram inadequados.

\footnotetext{
1 Universidade Federal da Paraíba, Centro de Ciências Agrárias, Av. Juarez Távora 1557, Expedicionários, 58040-021 João Pessoa, PB, Brasil

2 Universidade Federal da Paraíba, Centro de Ciências Agrárias, Laboratório de Ecologia Vegetal, 58397-000 Areia, PB, Brasil (landrade@cca.ufpb.br)

3 Universidade Federal da Paraíba, Laboratório de Botânica, 58397-000 Areia, PB, Brasil (lpfelix@ @otmail.com)

4 Universidade Federal de Lavras, Lavras, MG (imarinhopereira@gmail.com)

5 Autor para correspondência: (alvespegado@yahoo.com.br)
} 
Como decorrência dessa política, o bioma caatinga enfrenta atualmente mais um grave problema que é a invasão de algaroba - Prosopis juliflora (Sw) DC. Segundo Silva (1997), não se sabe a origem da P. juliflora, contudo, a espécie ocorre em regiões áridas e semi-áridas dos continentes Asiático, Africano e Americano. Esta espécie foi introduzida no semiárido nordestino no início da década de 40 (Gomes 1961; Azevedo 1982), tendo sido apresentada e difundida como uma promissora alternativa econômica, haja vista sua adaptação em diversas regiões semiáridas do mundo e por ser ela, uma espécie de uso múltiplo, produtora de lenha, madeira, forragem e outros produtos. Não obstante essas qualidades, a falta de manejo adequado, a adaptação regional da espécie, a facilidade de dispersão promovida pelos rebanhos, dentre outros fatores, transformaram em problema o que pretendia ser uma solução. A espécie foi sendo disseminada e se estabeleceu em determinados sítios da caatinga, ocupando grandes extensões de terras em praticamente todos os estados do Nordeste. Os sítios preferenciais da invasora são as áreas de matas ciliares, as manchas de Neossolos Flúvicos e as baixadas sedimentares, onde se formam maciços populacionais de alta densidade (L.A. Andrade, observação pessoal).

Ao contrário de muitos problemas ambientais que geralmente tendem a se amenizar com o decorrer do tempo, a contaminação biológica se multiplica ou se expande, causando impactos de longo prazo, não permitindo que os ecossistemas afetados se recuperem naturalmente (Westbrooks 1998). A invasão biológica é caracterizada quando um organismo ocupa, desordenadamente, um espaço fora de sua área de dispersão geográfica. É freqüentemente relacionada à influência do ser humano, intencional ou não, como também a processos naturais. Depois de estabelecido, o organismo, pode expandir-se aos habitats circunvizinhos, podendo ocasionar grandes perdas econômicas e/ou biológicas, pela extinção ou perturbação da biota nativa. As espécies invasoras geralmente afetam a estrutura das comunidades e/ou a funcionalidade dos ecossistemas (Williamson 1996). Segundo Parker et al. (1999) as invasões biológicas podem causar impactos em diversos níveis, incluindo efeitos sobre os indivíduos (morfologia, comportamento, mortalidade, crescimento), efeitos genéticos (alteração de padrões de fluxo gênico, hibridização), efeitos sobre a dinâmica de populações (abundância, crescimento populacional, extinção), sobre a comunidade (riqueza de espécies, diversidade, estrutura trófica) e sobre processos do ecossistema (disponibilidade de nutrientes, produtividade, regime de perturbações).

De acordo com Ziller \& Galvão (2002) e Randall (1996), a grande maioria dos países carece tanto de registros como de medidas de prevenção e controle de espécies invasoras, requerendo coleta e organização de dados para retratar a situação atual e para estabelecer prognósticos sobre o problema. Este seria exatamente o caso do Brasil, que sofre com o problema, mas que ainda não se conhece suas reais dimensões (S.R. Ziller, dados não publicados). Para a América do Sul, ainda existem poucos registros de plantas invasoras em ambientes florestais (Cronk \& Fuller 1995), provavelmente em decorrência de escassez de estudos com este enfoque direto (Pivello et al. 1999).

Em se tratando do bioma caatinga, é notória a escassez de informações científicas relativas até mesmo a abordagens mais básicas como, por exemplo, a sucessão ecológica, a estrutura fitossociológica das diferentes fisionomias, a dinâmica de regeneração, dentre outras. Em se tratando do tema invasão biológica, muito pouco foi estudado até o presente na caatinga, não obstante a gravidade do problema, particularmente causado por espécies introduzidas pelo homem, como foi o caso de P. juliflora. A caatinga vem sendo largamente substituída pelo sistema agropastoril, cujo modelo tem se revelado insustentável, causando sérios danos à ecologia e à sócio-economia de vastas áreas do interior nordestino, tais como o empobrecimento da biota, a degradação física dos solos e a desertificação (Brasil 1991; 2002; Sampaio et al. 2003).

Este estudo teve como objetivo avaliar os impactos causados pela invasão de $P$. juliflora sobre a composição e a estrutura do componente arbustivoarbóreo da caatinga, tanto no estrato adulto quanto na regeneração natural, em Monteiro, Paraíba. Pretende-se com isto ampliar os conhecimentos sobre o comportamento da caatinga afetada pelo problema em análise, subsidiar a ações conservacionistas e orientar políticas públicas voltadas para o controle de $P$. juliflora no referido bioma.

\section{Material e métodos}

O município de Monteiro está localizado na microrregião do Cariri Ocidental do Estado da Paraíba, ocupa uma área de $1.052 \mathrm{~km}^{2} \mathrm{e}$ apresenta uma altitude em torno de $600 \mathrm{~m}$. O clima é do tipo Bsh (semi-árido quente), segundo a classificação de Köppen, com 
precipitação pluviométrica média anual em torno dos $400 \mathrm{~mm}$. As médias de temperatura nunca são inferiores a $24{ }^{\circ} \mathrm{C}$ (Brasil 1971).

Para a realização deste trabalho foram selecionadas duas áreas de caatinga, assim descritas: Ambiente I = remanescente de caatinga arbórea em bom estado de conservação e sem a presença de P. juliflora. Situado às margens do rio Paraíba (753'69" S; 37 8'67" W), os solos predominantes são os Neossolos Flúvicos e a altitude local é de $621 \mathrm{~m}$. Este remanescente (30 ha), não sofreu corte raso pelo menos nos últimos 40 anos, embora haja relatos de que a área já tenha sido usada com agricultura, passando posteriormente a ser usada com pecuária extensiva, uso este que permanecia até à realização deste trabalho. O porte das árvores registradas no local valida o histórico levantado junto aos moradores das imediações. Ambiente II = Área de caatinga invadida (30 ha), por $P$. juliflora, contígua à área anterior e situada na mesma posição topográfica anteriormente descrita. Apresenta também os Neossolos Flúvicos como classe de solos predominante, haja vista que se localiza igualmente às margens do rio Paraíba (7053'36" S; 378'36" W), apresentando $615 \mathrm{~m}$ de altitude e, tal qual o Ambiente I, apresenta topografia plana. Esta área foi usada com agricultura durante mais de 40 anos e acerca de 20 anos foi abandonada quando, provavelmente, teve início a invasão de $P$. juliflora. Atualmente, a intensidade da invasão é variável de um ponto para outro, mas a espécie invasora já domina toda a área. Existem núcleos com indivíduos jovens e adultos altamente adensados e locais onde estes ocorrem em menor densidade, dividindo espaços com a pecuária extensiva de caprinos e bovinos.

Em cada ambiente foram distribuídas aleatoriamente 10 parcelas de $8 \times 50 \mathrm{~m}$, nas quais foi efetuado o inventário, tanto dos indivíduos adultos quanto dos regenerantes. Para efeito desta estratificação, considerou-se como adultos os indivíduos com diâmetro ao nível do solo (DNS) maior ou igual a $3 \mathrm{~cm}$ e altura maior ou igual a $1 \mathrm{~m}$ (Rodal et al. 1992), para o bioma caatinga. Como regenerantes, foram considerados todos os indivíduos que apresentavam diâmetro ao nível do solo (DNS) inferior a $3 \mathrm{~cm}$, independente da altura. Esta categoria de indivíduos foi hierarquizada nas seguintes classes de altura, conforme adaptação de Finol (1971): Classe I - indivíduos com altura variando de 0,10 a $0,30 \mathrm{~m}$; Classe II - indivíduos com altura $>0,30$ a 1,50 m; Classe III - indivíduos com altura $>1,5$ a 3,0 m e Classe IV - indivíduos com altura superior a 3,0 m, mas que apresentavam diâmetro ao nível do solo inferior a $3 \mathrm{~cm}$. A identificação das espécies foi feita com base em material fértil coletado e comparação com material de herbário.

Para descrever a estrutura das comunidades arbóreas, foram calculados os parâmetros fitossociológicos clássicos: densidades, frequiências e dominâncias absolutas e relativas, valor de importância e valor de cobertura (Curtis \& Macintosh 1951; Lamprecht 1962; 1964; Muller-Dombois \& Ellemberg 1974; Mateucci \& Colma 1982; Kent \& Coker 1999). Foram calculados, para cada ambiente, o índice de diversidade de Shannon, H' e a equabilidade de Pielou, J' (Krebs 1989) em base logarítmica natural (Brower \& Zar 1984). Os valores do índice de diversidade de Shannon dos dois ambientes foram comparados pelo teste de $t$ de Hutcheson (Zar 1996).

Foi realizada também, uma análise de agrupamento das vinte parcelas usadas neste estudo, baseando-se na composição de sua flora de adultos e regenerantes, com uso do programa PC-ORD, versão 4,0 (McCune \& Mefford 1999). A medida de similaridade florística empregada na análise de agrupamento foi o índice de Jaccard (Brower \& Zar 1984) e o método de agrupamento foi o das médias não ponderadas (UPGMA).

\section{Resultados e discussão}

Composição e Diversidade - Neste estudo foram inventariados, 971 indivíduos abustivo-arbóreos adultos e 1.233 indivíduos regenerantes. O Ambiente I apresentou 475 indivíduos adultos, pertencentes a 25 famílias, 29 gêneros e 35 espécies. No Ambiente II foram amostrados 496 indivíduos, pertencentes a 11 famílias, 16 gêneros e 16 espécies. Já para a categoria de regenerantes, foi registrada no Ambiente I a presença de 382 indivíduos, pertencentes a 18 famílias, 23 gêneros e 23 espécies. No Ambiente II foram inventariados 851 indivíduos, distribuídos em 13 famílias, 19 gêneros e 19 espécies.

Considerado apenas o Ambiente II, observou-se que, dos 496 indivíduos adultos ali registrados, 441 pertenciam à espécie $P$. juliflora, o que representa aproximadamente, $89 \%$ do total de indivíduos amostrados. Em relação aos regenerantes, a participação desta espécie no conjunto dos indivíduos desta categoria foi de, aproximadamente, $73 \%$, o que é uma densidade relativa também muito alta. Assim, constata-se uma dominância acentuada em relação à abundância de indivíduos, tanto para o estrato adulto 
como para os regenerantes. Diante destes resultados, fica evidente a agressividade de $P$. juliflora, a qual está caracterizada como uma espécie invasora que domina os ambientes colonizados e reduz drasticamente a participação das espécies nativas, seja eliminando-as por competição, seja impedindo que muitas delas se estabeleçam.

Em relação ao número de espécies, constata-se que o Ambiente I apresentou valores muito superiores àqueles encontrados no Ambiente II. Convém ressaltar que os números de espécies registrados em outros estudos realizados no bioma caatinga variam de 22 a 55 espécies (Araújo et al. 1995; Rodal et al. 1998; Ferraz et al. 1998; Drumond et al. 2002; Alcoforado Filho et al. 2003; Pereira et al. 2002). Estes resultados evidenciam que a presença de $P$. juliflora, interfere de forma severa, no processo de estabelecimento e de colonização das espécies nativas.

Das 49 espécies registradas nos dois Ambientes (Tab. 1), $23(48,97 \%)$ foram encontradas tanto no

Tabela 1. Relação das espécies arbóreas registradas nos dois Ambientes de caatinga, com e sem invasão de $P$. juliflora (Sw.) DC., no Município de Monteiro, PB. As espécies estão organizadas em ordem alfabética das famílias.

\begin{tabular}{|c|c|c|}
\hline Família / Espécie & Nome vulgar & Registro no herbário \\
\hline \multicolumn{3}{|l|}{ ANACARDIACEAE } \\
\hline 1. Schinopsis brasiliensis Engl. & baraúna & 9670 \\
\hline 2. Myracrodruon urundeuva Allem. & aroeira & 9669 \\
\hline \multicolumn{3}{|l|}{ APOCYNACEAE } \\
\hline 3. Aspidosperma pyrifolium Mart. & pereiro & 9712 \\
\hline \multicolumn{3}{|l|}{ ACANTACEAE } \\
\hline 4. Ruellia sp. & - & 9692 \\
\hline \multicolumn{3}{|l|}{ BORAGINACEAE } \\
\hline 5. Cordia globosa (Jacq.) H.B.F. & moleque-duro & 9706 \\
\hline \multicolumn{3}{|l|}{ CELASTRACEAE } \\
\hline 6. Maytenus rigida Mart. & bom-nome & 9671 \\
\hline \multicolumn{3}{|l|}{ COMBRETACEAE } \\
\hline 7. Combretum pisonioides Taub. & sipaúba & 9689 \\
\hline \multicolumn{3}{|l|}{ CACTACEAE } \\
\hline 8. Cereus jamacaru DC. & mandacaru & 9702 \\
\hline 9. Arrojadoa rhodantha (Guerke) Br. \& Rose & rabo-de-raposa & 9715 \\
\hline 10. Pilosocereus gounellei (Weber) Byl. \& Rowl. & xique-xique & 9716 \\
\hline \multicolumn{3}{|l|}{ CAESALPINACEAE } \\
\hline 11. Senna spectabilis (DC.) Irwin \& Barneby & canafístula & 9674 \\
\hline 12. Caesalpinia pyramidalis Tul. & catingueira & 9686 \\
\hline 13. Caesalpinia leiostachya (Benth.) Ducke & pau-ferro, jucá & 9677 \\
\hline 14. Caesalpinia ferrea Mart. ex Tul. & jucá & 9696 \\
\hline 15. Amburana cearensis (M. Allemão) A.C. Smith & pau-açú & 9711 \\
\hline \multicolumn{3}{|l|}{ CAPPARACEAE } \\
\hline 16. Cleome spinosa $\mathrm{L}$. & mussambê & 9707 \\
\hline 17. Capparis flexuosa $\mathrm{L}$. & feijão-bravo & 9683 \\
\hline \multicolumn{3}{|l|}{ EUPHORBIACEAE } \\
\hline 18. Jatropha pohliana Muell. Arg. & pinhão & 9713 \\
\hline 19. Croton sonderianus Muell. Arg. & marmeleiro & 9705 \\
\hline 20. Croton sp. 1 & - & 9678 \\
\hline \multicolumn{3}{|l|}{ ERYTHROXYLACEAE } \\
\hline 21. Erythroxylum simonis Plowman & guarda-orvalho, piranha & 9693 \\
\hline \multicolumn{3}{|l|}{ FLACOURTIACEAE } \\
\hline 22. Casearia guianensis Urb. & café-bravo & 9672 \\
\hline \multicolumn{3}{|l|}{ MYRTACEAE } \\
\hline 23. Myrcia sp. & canela-de-veado & 9675 \\
\hline \multicolumn{3}{|l|}{ MIMOSACEAE } \\
\hline 24. Anadenanthera colubrina (Vell.) Brenan & angico & 9667 \\
\hline 25. Prosopis juliflora (Sw.) D.C. & algaroba & 9665 \\
\hline 26. Chloroleucon foliolosum (Benth.) J.P. Lewis & jurema-branca & 9697 \\
\hline 27. Mimosa tenuiflora (Willd.) Poir & jurema-de-imbira & 9698 \\
\hline 28. Mimosa hostilis Benth. & jurema-preta & 9699 \\
\hline
\end{tabular}


Tabela 1 (continuação)

\begin{tabular}{|c|c|c|}
\hline Família / Espécie & Nome vulgar & Registro no herbário \\
\hline 29. Piptadenia zehntneri Harms & angico-monjolo & 9668 \\
\hline \multicolumn{3}{|l|}{ MALVACEAE } \\
\hline 30. Abutilon sp. & - & 9676 \\
\hline 31. Malvestarum scaberum Jaccke. & - & 9691 \\
\hline \multicolumn{3}{|l|}{ PAPILONACEAE } \\
\hline 32. Lonchocarpus campestris Benth. & rabo-de-cavalo & 9690 \\
\hline 33. Erythrina velutina Willd. & mulungu & 9708 \\
\hline 34. Indigofera suffruticiosa Mill. & anil & 9666 \\
\hline \multicolumn{3}{|l|}{ RUBIACEAE } \\
\hline 35. Tocoyena formosa Schum. & jenipapo-bravo & 9694 \\
\hline 36. Guettarda platypoda DC. & angélica & 9679 \\
\hline \multicolumn{3}{|l|}{ RHAMNACEAE } \\
\hline 37. Zizyphus joazeiro Mart. & juazeiro & 9695 \\
\hline 38. Rhamnidium molle Reissek. & - & 9681 \\
\hline \multicolumn{3}{|l|}{ RUTACEAE } \\
\hline 39. Zanthoxylum hiemale A. St.-Hil. & limão-bravo & 9701 \\
\hline \multicolumn{3}{|l|}{ SAPOTACEAE } \\
\hline 40. Sideroxylon obtusifolium (Roem. \& Schult.) Penn. & quixabeira & 9714 \\
\hline \multicolumn{3}{|l|}{ STERCULIACEAE } \\
\hline 41. Helicteris erchleri $\mathrm{K}$. Schum. & - & 9682 \\
\hline 42. Melochia tomentosa $\mathrm{L}$. & maria-preta & 9704 \\
\hline \multicolumn{3}{|l|}{ SOLANACEAE } \\
\hline 43. Solanum paniculatum $\mathrm{L}$. & jurubeba & 9700 \\
\hline 44. Nicotiana glauca Grah. & oliveira & 9710 \\
\hline \multicolumn{3}{|l|}{ VERBENACEAE } \\
\hline 45. Lantana camara $\mathrm{L}$. & camará, chumbinho & 9673 \\
\hline 46. Vitex gardnerianum Schauer & - & 9680 \\
\hline 47. indeterminada 1 & chorão & 9685 \\
\hline 48. indeterminada 2 & pau-caixão & 9688 \\
\hline 49. indeterminada 3 & - & 9717 \\
\hline
\end{tabular}

estrato adulto, quanto na regeneração. Estas espécies são as seguintes: Arrojadoa rhodantha, Anadenanthera colubrina, Cordia globosa, Combretum pisonioides, Cereus jamacaru, Capparis flexuosa, Caesalpinia pyramidalis, Caesalpinia ferrea, Croton sonderianus, Casearia guianensis, Jatropha pohliana, Lantana camara, Mimosa hostilis, Nicotiana glauca, Prosopis juliflora, Ruellia sp., Rhamnidium molle, Schinopsis brasiliensis, Senna spectabilis, Sideroxylon obtusifolium, Solanum paniculatum, Zanthoxylum hiemale e Zizyphus joazeiro.

Embora situadas muito próximas e em condições ambientais análogas, os dois Ambientes apresentaram-se como conjuntos distintos, tanto em relação à composição de adultos, quanto à regeneração natural, conforme revelou a análise de agrupamento. O dendrograma de similaridade mostrou claramente a formação de dois grandes grupos: o primeiro formado pelas parcelas 1A a 10A, corresponde ao Ambiente I e o outro, formado pelas parcelas $1 \mathrm{~B}$ a 10B, correspondente ao Ambiente II (Fig. 1).

Os resultados mostram que tanto a regeneração natural quanto o componente adulto do Ambiente II, apresentaram composição florística muito pobre, o que se explica pela grande abundância da $P$. juliflora. A proliferação de $P$. juliflora na caatinga é uma constatação, porém as consequiências desse problema não são ainda de todo previsíveis, haja vista que a invasão biológica é um tema que só recentemente começou a ser estudado. As características que aumentam as chances de uma espécie se tornar invasora, bem como a suscetibilidade ambiental à invasão biológica são abordagens que praticamente começaram a ser estudadas na última década (Noble 1989; Roy 1990; Rejmanek \& Richardson 1996; Williamson \& Fitter 1996).

$O$ valor de diversidade para a caatinga sem a presença de $P$. juliflora (Ambiente I), para o componente adulto $\left(\mathrm{H}^{\prime}=2,81\right)$ foi significativamente superior ao encontrado para a caatinga com invasão de $P$. juliflora (Ambiente II) $\left(\mathrm{H}^{\prime}=0,61 ; \mathrm{P}<0,001\right)$. 


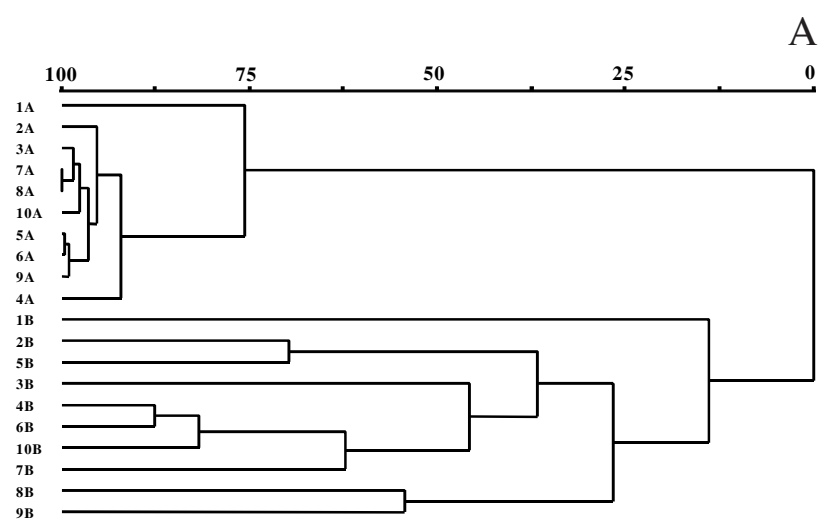

B

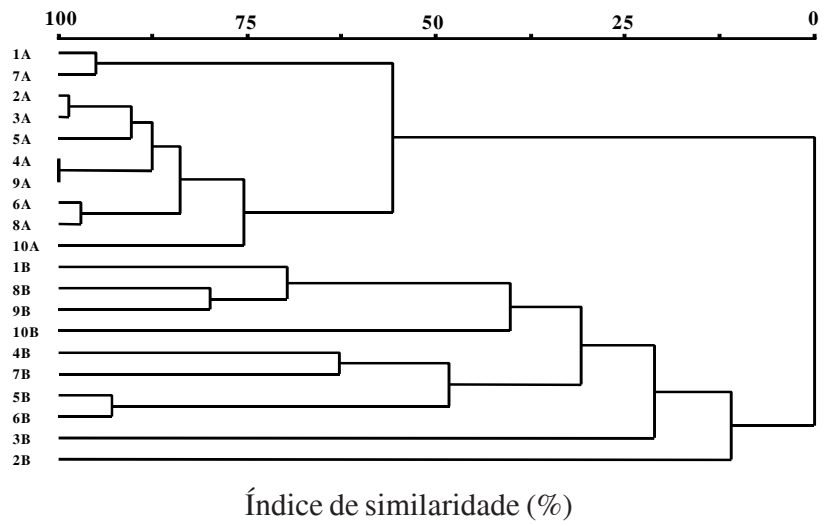

Figura 1. Dendrograma de similaridade produzido por análise de agrupamento pelo método UPGMA com base no índice de Jaccard, para os dados de presença e ausência das espécies em 20 parcelas instaladas em dois Ambientes de caatinga no Município de Monteiro, PB. A = Estrato Arbustivo-arbóreo; B = Regeneração Natural; $1 \mathrm{~A}$ a $10 \mathrm{~A}=$ parcelas da caatinga invadida por $P$. juliflora (Sw.) DC.; 1B a 10B = parcelas da caatinga sem a presença de P. juliflora.

Para a regeneração natural o valor de H', para a caatinga não invadida foi de 2,31 e para a caatinga com invasão de P. juliflora, 1,14 ( $\mathrm{P}<0,001)$. Os valores de equabilidade foram altos na caatinga sem $P$. juliflora, tanto para a fase adulta $\left(\mathrm{J}^{\prime}=0,791\right)$, quanto para a regeneração natural $\left(\mathrm{J}^{\prime}=0,738\right)$, e baixos para a caatinga invadida ( $\mathrm{J}^{\prime}=0,219$ e 0,389$)$ para adultos e regenerantes, respectivamente. Estes resultados evidenciam a dominância ecológica da espécie exótica e mostram que grande parte das espécies nativas, mesmo se estabelecendo, não consegue atingir a idade adulta, o que se reflete em uma pobreza ainda maior naquele componente. Considerando que o Ambiente I é fisicamente igual ao Ambiente II, os baixos índices de diversidade encontrados neste último são justificados, em grande parte pela invasão de $P$. juliflora, cuja presença maciça impediu o estabelecimento de muitas espécies autóctones, interferindo negativamente no processo de sucessão ecológica. Quando se analisa os dados de diversidade obtidos no Ambiente I, constata-se que estes valores estão próximos daqueles encontrados em outros estudos realizados em remanescentes de caatinga com histórico similar, cujas grandezas variaram de 1,91 a 2,99 (Araújo et al. 1995; Ferraz et al. 1998; Rodal et al. 1998; Pereira et al. 2002). Situação diferente ocorre com a área invadida por $P$. juliflora, pois mesmo decorridos mais 20 anos de abandono ainda apresenta diversidade muito baixa, incompatível com os valores registrados para a caatinga, além de estar ocupada pela invasora cuja densidade relativa chega a quase $90 \%$. Isto mostra que a presença desta espécie interfere no processo de restauração da diversidade biológica dos campos abandonados.

Estes resultados são confirmados pelo estudo realizado por Shiferaw et al. (2004) sobre as características biológicas que promovem a invasão da P. juliflora no Nordeste da Etiópia. Os autores constataram que essa espécie apresenta características biológicas que a tornam uma invasora potencial, capaz de ocupar muito rapidamente novas áreas, até mesmo sob condições áridas. Os autores ressaltam as seguintes estratégias importantes da espécie: alta produção de sementes, produção de frutos atrativos para os animais, capacidade de sobrevivência dos propágulos à digestão dos animais; formação de banco de sementes no solo; acúmulo de reservas que viabilizam a dormência e a longevidade das sementes que servirão como estoque para a regeneração; início precoce da fase reprodutiva; rápido crescimento no tempo e no espaço, dentre outras. Este conjunto de características, muitas das quais observadas pelos autores deste trabalho, tornam $P$. juliflora uma espécie invasora, caráter este que pode ser potencializado por fatores antrópicos, físicos, ecológicos e ambientais, a exemplo do que está ocorrendo no semi-árido nordestino.

Estrutura - No Ambiente I, foi registrada uma densidade de 1.188 indivíduos ha ${ }^{-1}$, na fase adulta e de 955 indivíduos ha-1 ${ }^{-1}$ para a regeneração natural (Tab. 2 e 3). Para o Ambiente II, constatou-se uma densidade de 1.240 indivíduos $\mathrm{ha}^{-1}$, para os adultos e de 2.128 indivíduos $\mathrm{ha}^{-1}$, para a regeneração natural (Tab. 4 e 5), o que demonstra a agressividade da invasora.

O parâmetro área basal expressa o crescimento e a produção de biomassa nos ecossistemas florestais (Scolforo \& Figueiredo Filho 1998). Verifica-se para o 
Tabela 2. Parâmetros quantitativos e estruturais do estrato arbustivo-arbóreo de um Ambiente de caatinga sem a presença de $P$. juliflora (Sw.) DC. em Monteiro, PB (Ambiente I). NI = número de indivíduos; $\mathrm{P}=$ número de parcelas com ocorrência da espécie; $\mathrm{DA}=$ densidade absoluta (indivíduos ha $\left.{ }^{-1}\right) ; \mathrm{DR}=$ densidade relativa $(\%) ; \mathrm{AB}=$ área basal $\left(\mathrm{m}^{2} \mathrm{ha}^{-1}\right) ; \mathrm{ABR}=$ área basal relativa $(\%)$; $\mathrm{VC}=$ valor de cobertura; $\mathrm{VI}=$ valor de importância.

\begin{tabular}{|c|c|c|c|c|c|c|c|c|}
\hline \multirow[t]{2}{*}{ Espécie } & \multicolumn{8}{|c|}{ Estrato arbóreo } \\
\hline & NI & $\mathrm{P}$ & DA & DR & $\mathrm{AB}$ & $\mathrm{ABR}$ & $\mathrm{VC}$ & VI \\
\hline Zizyphus joazeiro & 56 & 10 & 140,0 & 11,79 & 5,3423 & 17,91 & 14,80 & 12,74 \\
\hline Erythrina velutina & 21 & 6 & 52,5 & 4,42 & 6,7498 & 22,62 & 13,50 & 10,72 \\
\hline Caesalpinia pyramidalis & 54 & 9 & 135,0 & 11,37 & 2,8217 & 9,46 & 10,40 & 9,50 \\
\hline Lonchocarpus campestris & 74 & 3 & 185,0 & 15,58 & 1,8566 & 6,22 & 10,90 & 8,12 \\
\hline Sideroxylon obtusifolium & 47 & 8 & 117,5 & 9,89 & 1,9187 & 6,43 & 8,16 & 7,72 \\
\hline Anadenanthera colubrina & 26 & 4 & 65,0 & 5,47 & 3,2327 & 10,84 & 8,15 & 6,57 \\
\hline Schinopsis brasiliensis & 16 & 6 & 40,0 & 3,37 & 2,2495 & 7,54 & 5,45 & 5,34 \\
\hline Capparis flexuosa & 34 & 8 & 85,0 & 7,16 & 0,5178 & 1,74 & 4,45 & 5,24 \\
\hline Myrcia sp. & 38 & 5 & 95,0 & 8,00 & 0,8747 & 2,93 & 5,47 & 5,07 \\
\hline Zanthoxylum hiemale & 5 & 3 & 12,5 & 1,05 & 0,0807 & 0,27 & 0,66 & 3,89 \\
\hline Croton sp. & 12 & 4 & 30,0 & 2,53 & 15,331 & 5,14 & 3,83 & 3,69 \\
\hline Croton sonderianus & 16 & 5 & 40,0 & 3,37 & 0,3654 & 1,22 & 2,30 & 2,95 \\
\hline Cereus jamacaru & 12 & 5 & 30,0 & 2,53 & 0,1731 & 0,58 & 1,55 & 2,46 \\
\hline Erythroxylum simonis & 9 & 5 & 22,5 & 1,89 & 0,0926 & 0,31 & 1,10 & 2,16 \\
\hline Indeterminada 2 & 7 & 3 & 17,5 & 1,47 & 0,7221 & 2,42 & 1,95 & 2,15 \\
\hline Jatropha pohliana & 6 & 5 & 15,0 & 1,26 & 0,0139 & 0,05 & 0,65 & 1,86 \\
\hline Indeterminada 1 & 5 & 3 & 12,5 & 1,05 & 0,1861 & 0,62 & 0,84 & 1,41 \\
\hline Rhaminidium molle & 8 & 2 & 20,0 & 1,68 & 0,172 & 0,58 & 1,13 & 1,31 \\
\hline Senna spectabilis & 4 & 3 & 10,0 & 0,84 & 0,0129 & 0,04 & 0,44 & 1,15 \\
\hline Caesalpinia ferrea & 2 & 2 & 5,0 & 0,42 & 0,284 & 0,95 & 0,69 & 1,02 \\
\hline Maytenus rigida & 4 & 2 & 10,0 & 0,84 & 0,08 & 0,27 & 0,56 & 0,94 \\
\hline Aspidosperma pyrifolium & 2 & 2 & 5,0 & 0,42 & 0,0863 & 0,29 & 0,36 & 0,80 \\
\hline Mimosa tenuiflora & 2 & 2 & 5,0 & 0,42 & 0,0245 & 0,08 & 0,25 & 0,73 \\
\hline Caesalpinia leiostachya & 1 & 1 & 2,5 & 0,21 & 0,1827 & 0,61 & 0,41 & 0,56 \\
\hline Morto & 3 & 1 & 7,5 & 0,63 & 0,014 & 0,05 & 0,34 & 0,51 \\
\hline Amburana cearensis & 1 & 1 & 2,5 & 0,21 & 0,1112 & 0,37 & 0,29 & 0,48 \\
\hline Tocoyena formosa & 2 & 1 & 5,0 & 0,42 & 0,022 & 0,07 & 0,25 & 0,45 \\
\hline Casearia guianensis & 1 & 1 & 2,5 & 0,21 & 0,0307 & 0,10 & 0,16 & 0,39 \\
\hline Helicteris erchleri & 1 & 1 & 2,5 & 0,21 & 0,0255 & 0,09 & 0,15 & 0,38 \\
\hline Chloroleucon foliolosum & 1 & 1 & 2,5 & 0,21 & 0,0238 & 0,08 & 0,15 & 0,38 \\
\hline Combretum psisonioides & 1 & 1 & 2,5 & 0,21 & 0,0196 & 0,07 & 0,14 & 0,37 \\
\hline Vitex gardnerianum & 1 & 1 & 2,5 & 0,21 & 0,0059 & 0,02 & 0,12 & 0,36 \\
\hline Solanum paniculatum & 1 & 1 & 2,5 & 0,21 & 0,0031 & 0,01 & 0,11 & 0,36 \\
\hline Ruellia sp. & 1 & 1 & 2,5 & 0,21 & 0,0031 & 0,01 & 0,11 & 0,36 \\
\hline Cordia globosa & 1 & 1 & 2,5 & 0,21 & 0,0018 & 0,01 & 0,11 & 0,35 \\
\hline Total & 475 & 10 & 1.188 & 100 & 29,83 & 100 & 100 & 100 \\
\hline
\end{tabular}

Ambiente I (Tab. 2), um valor de aproximadamente, $30 \mathrm{~m}^{2} \mathrm{ha}^{-1}$, para a vegetação adulta, com desataque para as espécies Erythrina velutina, Ziziphus joazeiro, Anadenanthera columbrina, Caesalpinia pyramidalis, Schinopsis brasiliensis e Sideroxylon obtusifolium, que juntas representaram, aproximadamente, $75 \%$ da área basal total. Já para a regeneração natural (Tab. 3), obteve-se uma área basal de $1,8 \mathrm{~m}^{2} \mathrm{ha}^{-1}$. Neste componente as espécies que mais se destacaram foram: Croton sonderianus, Ziziphus joazeiro, Caesalpinia pyramidalis, Cordia globosa e Jatropha polhiana, juntas representaram, aproximadamente, $75 \%$ do valor total.

No Ambiente II a área basal total foi de $9,15 \mathrm{~m}^{2} \mathrm{ha}^{-1}$, para a fase adulta e de $4,5 \mathrm{~m}^{2} \mathrm{ha}^{-1}$, para a regeneração (Tab. 4 e 5). Comparando-se esses valores entre as duas situações constata-se que houve uma grande redução dos mesmos, tanto para adultos quanto para regenerantes. Uma das principais características das plantas invasoras é formar maciços populacionais, cujo adensamento acaba retardando o crescimento de grande parte dos indivíduos, o que 
Tabela 3. Parâmetros quantitativos e estruturais da regeneração natural de um Ambiente de caatinga sem a presença de $P$. juliflora (Sw.) DC. em Monteiro, PB (Ambiente I). NI = número de indivíduos; $\mathrm{P}=$ número de parcelas com ocorrência da espécie; $\mathrm{DA}=$ densidade absoluta (indivíduos ha $\left.{ }^{-1}\right) ; \mathrm{DR}=$ densidade relativa $(\%) ; \mathrm{AB}=$ área basal $\left(\mathrm{m}^{2} \mathrm{ha}^{-1}\right) ; \mathrm{ABR}=$ área basal relativa $(\%)$; VC= valor de cobertura; VI = valor de importância.

\begin{tabular}{|c|c|c|c|c|c|c|c|c|}
\hline \multirow[t]{2}{*}{ Espécie } & \multicolumn{8}{|c|}{ Regeneração natural } \\
\hline & NI & $\mathrm{P}$ & $\mathrm{DA}$ & DR & $\mathrm{AB}$ & $\mathrm{ABR}$ & $\mathrm{VC}$ & VI \\
\hline Solanum paniculatum & 100 & 4 & 250,0 & 26,18 & 0,0877 & 7,47 & 16,82 & 13,04 \\
\hline Croton sonderianus & 51 & 4 & 127,5 & 13,35 & 0,2245 & 19,13 & 16,24 & 12,65 \\
\hline Caesalpinia pyramidalis & 46 & 9 & 115,0 & 12,04 & 0,1509 & 12,86 & 12,45 & 12,41 \\
\hline Cordia globosa & 51 & 7 & 127,5 & 13,35 & 0,1446 & 12,32 & 12,84 & 11,75 \\
\hline Zizyphus joazeiro & 42 & 6 & 105,0 & 10,99 & 0,1594 & 13,59 & 12,29 & 10,93 \\
\hline Jatropha pohliana & 11 & 6 & 27,5 & 2,88 & 0,0826 & 7,04 & 4,96 & 6,04 \\
\hline Cereus jamacaru & 14 & 4 & 35,0 & 3,66 & 0,0765 & 6,52 & 5,09 & 5,22 \\
\hline Rhaminidium molle & 12 & 3 & 30,0 & 3,14 & 0,0619 & 5,28 & 4,21 & 4,17 \\
\hline Zanthoxylum hiemale & 17 & 5 & 42,5 & 4,45 & 0,0105 & 0,89 & 2,67 & 4,06 \\
\hline Guettarda platypoda & 8 & 3 & 20,0 & 2,09 & 0,0378 & 3,22 & 2,66 & 3,14 \\
\hline Senna spectabilis & 7 & 4 & 17,5 & 1,83 & 0,0181 & 1,54 & 1,69 & 2,95 \\
\hline Lantana camara & 6 & 4 & 15,0 & 1,57 & 0,0094 & 0,80 & 1,19 & 2,61 \\
\hline Sideroxylon obtusifolium & 4 & 2 & 10,0 & 1,05 & 0,0347 & 2,96 & 2,00 & 2,24 \\
\hline Arrojadoa rhodantha & 2 & 2 & 5,0 & 0,52 & 0,0215 & 1,83 & 1,18 & 1,70 \\
\hline Capparis flexuosa & 2 & 2 & 5,0 & 0,52 & 0,0095 & 0,81 & 0,67 & 1,36 \\
\hline Abutilon sp. & 2 & 1 & 5,0 & 0,52 & 0,0108 & 0,92 & 0,72 & 0,93 \\
\hline Ruellia sp. & 1 & 1 & 2,5 & 0,26 & 0,0095 & 0,81 & 0,54 & 0,81 \\
\hline Erythroxylum simonis & 1 & 1 & 2,5 & 0,26 & 0,0064 & 0,55 & 0,40 & 0,72 \\
\hline Anadenanthera colubrina & 1 & 1 & 2,5 & 0,26 & 0,0050 & 0,43 & 0,35 & 0,68 \\
\hline Combretum psisonioides & 1 & 1 & 2,5 & 0,26 & 0,0044 & 0,38 & 0,32 & 0,67 \\
\hline Desconhecida 2 & 1 & 1 & 2,5 & 0,26 & 0,0044 & 0,38 & 0,32 & 0,67 \\
\hline Malvestarum scaberum & 1 & 1 & 2,5 & 0,26 & 0,0028 & 0,24 & 0,25 & 0,62 \\
\hline Indeterminada 3 & 1 & 1 & 2,5 & 0,26 & 0,0005 & 0,04 & 0,15 & 0,55 \\
\hline Total & 382 & 10 & 955 & 100 & 1,173 & 100 & 100 & 100 \\
\hline
\end{tabular}

Tabela 4. Parâmetros quantitativos e estruturais do estrato arbustivo-arbóreo de um Ambiente de caatinga invadido por P. juliflora (Sw.) DC. no município de Monteiro, PB (Ambiente II). NI = número de indivíduos; $\mathrm{P}=$ número de parcelas com ocorrência da espécie; $\mathrm{DA}=$ densidade absoluta (indivíduos ha $\left.{ }^{-1}\right) ; \mathrm{DR}=$ densidade relativa $(\%) ; \mathrm{AB}=$ área basal $\left(\mathrm{m}^{2} \mathrm{ha}^{-1}\right) ; \mathrm{ABR}=$ área basal relativa $(\%) ;$ $\mathrm{VC}=$ valor de cobertura; $\mathrm{VI}=$ valor de importância.

\begin{tabular}{|c|c|c|c|c|c|c|c|c|}
\hline \multirow[t]{2}{*}{ Espécie } & \multicolumn{8}{|c|}{ Estrato arbóreo } \\
\hline & NI & $\mathrm{P}$ & $\mathrm{DA}$ & DR & $\mathrm{AB}$ & $\mathrm{ABR}$ & $\mathrm{VC}$ & VI \\
\hline Prosopis juliflora & 441 & 10 & 1102,5 & 88,91 & 8,6693 & 94,79 & 91,85 & 70,75 \\
\hline Cereus jamacaru & 11 & 5 & 27,5 & 2,22 & 0,1200 & 1,31 & 1,77 & 5,94 \\
\hline Arrojadoa rhodantha & 9 & 3 & 22,5 & 1,81 & 0,0290 & 0,32 & 1,07 & 3,56 \\
\hline Sideroxylon obtusifolium & 8 & 2 & 20,0 & 1,61 & 0,0928 & 1,01 & 1,31 & 2,78 \\
\hline Mimosa hostilis & 7 & 2 & 17,5 & 1,41 & 0,0362 & 0,40 & 0,90 & 2,50 \\
\hline Zizyphus joazeiro & 2 & 2 & 5,0 & 0,40 & 0,0057 & 0,06 & 0,23 & 2,06 \\
\hline Lantana camara & 2 & 2 & 5,0 & 0,40 & 0,0035 & 0,04 & 0,22 & 2,05 \\
\hline Jatropha pohliana & 3 & 1 & 7,5 & 0,60 & 0,1165 & 1,27 & 0,94 & 1,58 \\
\hline Capparis flexuosa & 4 & 1 & 10,0 & 0,81 & 0,0142 & 0,16 & 0,48 & 1,27 \\
\hline Solanum paniculatum & 3 & 1 & 7,5 & 0,60 & 0,0111 & 0,12 & 0,36 & 1,19 \\
\hline Nicotiana glauca & 1 & 1 & 2,5 & 0,20 & 0,0204 & 0,22 & 0,21 & 1,09 \\
\hline Schinopsis brasiliensis & 1 & 1 & 2,5 & 0,20 & 0,0126 & 0,14 & 0,17 & 1,06 \\
\hline Anadenanthera colubrina & 1 & 1 & 2,5 & 0,20 & 0,0053 & 0,06 & 0,13 & 1,04 \\
\hline Casearia guianensis & 1 & 1 & 2,5 & 0,20 & 0,0024 & 0,03 & 0,11 & 1,03 \\
\hline Combretum psisonioides & 1 & 1 & 2,5 & 0,20 & 0,0031 & 0,03 & 0,12 & 1,03 \\
\hline Pilosocereus gounellei & 1 & 1 & 2,5 & 0,20 & 0,0040 & 0,04 & 0,12 & 1,03 \\
\hline Total & 496 & 10 & 1.240 & 100 & 9,146 & 100 & 100 & 100 \\
\hline
\end{tabular}


Tabela 5. Parâmetros quantitativos e estruturais da regeneração natural de um Ambiente de caatinga invadido por $P$. juliflora (Sw.) DC. no Município de Monteiro, PB (Ambiente II). NI = número de indivíduos; $\mathrm{P}=$ número de parcelas com ocorrência da espécie; $\mathrm{DA}=$ densidade absoluta (indivíduos ha $\left.\mathrm{h}^{-1}\right) ; \mathrm{DR}=$ densidade relativa $(\%) ; \mathrm{AB}=$ área basal $\left(\mathrm{m}^{2} \mathrm{ha}^{-1}\right) ; \mathrm{ABR}=$ área basal relativa $(\%)$; $\mathrm{VC}=$ valor de cobertura; $\mathrm{VI}=$ valor de importância.

\begin{tabular}{|c|c|c|c|c|c|c|c|c|}
\hline \multirow[t]{2}{*}{ Espécie } & \multicolumn{8}{|c|}{ Regeneração natural } \\
\hline & NI & $\mathrm{P}$ & DA & DR & $\mathrm{AB}$ & $\mathrm{ABR}$ & $\mathrm{VC}$ & VI \\
\hline Prosopis juliflora & 646 & 10 & 1615,0 & 75,91 & 3,6003 & 80,30 & 78,11 & 59,01 \\
\hline Arrojadoa rhodantha & 23 & 6 & 57,5 & 2,70 & 0,2028 & 4,52 & 3,61 & 6,57 \\
\hline Cereus jamacaru & 16 & 5 & 40,0 & 1,88 & 0,1049 & 2,34 & 2,11 & 4,88 \\
\hline Indigofera suffruticosa & 34 & 4 & 85,0 & 4,00 & 0,0438 & 0,98 & 2,49 & 4,43 \\
\hline Mimosa hostilis & 21 & 4 & 52,5 & 2,47 & 0,1009 & 2,25 & 2,36 & 4,35 \\
\hline Lantana camara & 15 & 3 & 37,5 & 1,76 & 0,0562 & 1,25 & 1,51 & 3,09 \\
\hline Solanum paniculatum & 31 & 1 & 77,5 & 3,64 & 0,1170 & 2,61 & 3,13 & 2,78 \\
\hline Jatropha pohliana & 20 & 2 & 50,0 & 2,35 & 0,0695 & 1,55 & 1,95 & 2,69 \\
\hline Myracrodruon urundeuva & 4 & 2 & 10,0 & 0,47 & 0,0101 & 0,23 & 0,35 & 1,62 \\
\hline Zizyphus joazeiro & 3 & 2 & 7,5 & 0,35 & 0,0151 & 0,34 & 0,34 & 1,62 \\
\hline Caesalpinia ferrea & 14 & 1 & 35,0 & 1,65 & 0,0184 & 0,41 & 1,03 & 1,38 \\
\hline Casearia guianensis & 7 & 1 & 17,5 & 0,82 & 0,0476 & 1,06 & 0,94 & 1,32 \\
\hline Nicotiana glauca & 4 & 1 & 10,0 & 0,47 & 0,0483 & 1,08 & 0,77 & 1,21 \\
\hline Combretum psisonioides & 4 & 1 & 10,0 & 0,47 & 0,0147 & 0,33 & 0,40 & 0,96 \\
\hline Cleome spinosa & 3 & 1 & 7,5 & 0,35 & 0,0177 & 0,39 & 0,37 & 0,94 \\
\hline Capparis flexuosa & 3 & 1 & 7,5 & 0,35 & 0,0006 & 0,01 & 0,18 & 0,81 \\
\hline Schinopsis brasiliensis & 1 & 1 & 2,5 & 0,12 & 0,0095 & 0,21 & 0,16 & 0,80 \\
\hline Piptadenia zehntneri & 1 & 1 & 2,5 & 0,12 & 0,0044 & 0,10 & 0,11 & 0,76 \\
\hline Meloctia tomentosa & 1 & 1 & 2,5 & 0,12 & 0,0016 & 0,04 & 0,08 & 0,74 \\
\hline Total & 851 & 10 & 2.128 & 100 & 4,483 & 100 & 100 & 100 \\
\hline
\end{tabular}

concorre para o acréscimo do número de regenerantes. Some-se a isto, também, a capacidade de multiplicação dessas espécies, alem das vantagens competitivas que elas apresentam (Ziller \& Galvão 2002). Neste trabalho foi constatada uma dominância de $P$. juliflora para os dois componentes do Ambiente invadido, de modo que esta espécie foi responsável por $95 \%$ da área basal total do componente adulto e de $80 \%$ da regeneração natural.

Em relação ao valor de cobertura (VC) para a fase adulta, no Ambiente I, observa-se na Tab. 2, que espécies como Ziziphus joazeiro, Erythrina velutina, Lonchocarpus campestris, Caesalpinia pyramidalis, e Sideroxylon obtusifolium se destacaram em relação às demais. Juntas estas espécies apresentaram aproximadamente, $58 \%$ do VC total. No entanto, no Ambiente II, uma única espécie ( $P$. juliflora) apresentou valor de cobertura de aproximadamente, $92 \%$, o que caracteriza mais uma vez a dominância desta espécie em relação às demais (Tab. 4).

No que se refere à regeneração natural, no Ambiente I, espécies como Solanum paniculatum (16,82), Croton sonderianus (16,24), Caesalpinia pyramidalis $(12,45)$, Cordia globosa $(12,84)$ e Ziziphus joazeiro $(12,29)$, se destacaram em relação aos valores de cobertura (VC), no entanto, verifica-se na Tab. 3, que de modo geral, não há uma grande discrepância entre estes valores para as cinco espécies citadas, as quais respondem juntas por aproximadamente $70 \%$ do VC total. Por outro lado, no Ambiente II, apenas a espécie P. juliflora totalizou, aproximadamente, $78 \%$ do valor total (Tab. 5), o que confirma as afirmações aqui apresentadas.

Com relação ao Valor de Importância (VI), na regeneração Tab. 3, verifica-se que no Ambiente I foram as seguintes espécies que apresentaram maiores valores, Solanum paniculatum $(13,04)$, Croton sonderianus $(12,65)$, Caesalpinia pyramidalis $(12,41)$, Cordia globosa $(11,75)$ e Ziziphus joazeiro $(10,93)$; já no Ambiente II (Tab. 5), as espécies com maiores valores de VI foram P. juliflora $(59,01)$; Arrojadoa rhodantha (6,57), Cereus jamacaru $(4,88)$, Indigofera suffruticosa (4,43) e Mimosa hostilis $(4,35)$; com relação à fase adulta no Ambiente I, a espécie Ziziphus joazeiro apresentou o VI mais alto 12,77 enquanto as Erythrina velutina, Caesalpinia pyramidalis, Lonchocarpus campestris e Sideroxylon obtusifolium foram 10,74; 9,53; 8,28 e 7,74 respectivamente (Tab. 2). No Ambiente II, a espécie $P$. juliflora apresentou o VI mais alto, 70,76 
enquanto Cereus jamacaru, Arrojadoa rhodantha, Sideroxylon obtusifolium e Mimosa hostilis apresentaram os valores 5,$94 ; 3,57 ; 2,78$ e 2,51 , respectivamente (Tab. 4).

Randall (1996) relata que invasoras prejudiciais são aquelas que causam grandes modificações na riqueza, na abundância das espécies nativas e que podem destruir total ou parcialmente um ecossistema. Os resultados deste trabalho confirmam que $P$. juliflora se enquadra nesta categoria, pois os impactos detectados, quando da sua ocorrência nas comunidades autóctones, apontam exatamente nesta direção. Um dos fatores que contribuiu e continua contribuindo para essa dispersão da invasora é a ação antrópica acentuada. O homem vem provocando o desmatamento e expandindo a agricultura e a pecuária contribuindo para a o estabelecimento e invasão da P. juliflora na região.

Embora ainda não exista um consenso para uma abordagem de quantificação dos impactos de invasões biológicas, Parker et al. (1999) sugerem que três fatores devem ser levados em conta para medi-lo: área total ocupada, abundância local e a velocidade de invasão. Quanto maiores forem esses fatores, maiores serão os impactos causados pela espécie invasora. O raciocínio utilizado no uso da abundância do invasor como medida do impacto é de que qualquer biomassa (ou espaço, ou energia) controlada pelo invasor representa recursos não mais disponíveis para competidores (Parker et al. 1999).

A participação da $P$. juliflora alterou a fitodiversidade do Ambiente invadido, a ponto de torná-la um conjunto distinto, quanto à composição florística, à diversidade e à estrutura. Além dos indivíduos adultos funcionarem ativamente como fontes de propágulos para a invasão, os rebanhos criados na região funcionam como facilitadores, aliados ao homem que explora a caatinga, tornando-a aberta e, portanto vulnerável à invasão. $\mathrm{O}$ presente estudo evidencia a necessidade de se desenvolver ações conservacionistas e políticas de controle de $P$. juliflora, ações estas que passam pelo controle físico da invasora, mas certamente exigirá também mudanças nos costumes locais e no manejo dos rebanhos. Neste particular, faz-se ainda necessário estudar o real papel dos rebanhos como dispersores, haja vista que não se conhece as respostas fisiológicas das sementes à ingestão por diferentes animais.

Distribuição dos indivíduos em classes de diâmetro Na Fig. 2A, observa-se que, para o componente adulto, houve uma maior concentração de indivíduos na classe de 5 a $8 \mathrm{~m}$ de altura no Ambiente I, sendo que dos 165 indivíduos registrados nesta classe de altura 45, $(27,27 \%)$ pertenciam à Lonchocarpus campestris. Já para o Ambiente II, verifica-se uma maior ocorrência de indivíduos na classe de 3 a $5 \mathrm{~m}$ de altura, sendo que dos 205 indivíduos, 190 (92,68\%) eram de P. juliflora. O Ambiente I apresentou um padrão de distribuição de indivíduos nas classes de altura semelhante ao registrado por Pereira et al. (2002) em remanescentes de caatinga no agreste paraibano. Já para a distribuição das espécies nas classes de altura no componente regeneração natural (Fig. 2B), verifica-se que a maior densidade foi registrada na classe 0,30 a $1,50 \mathrm{~m}$ de altura para as duas situações estudadas. Vale salientar que dos 472 indivíduos registrados no Ambiente II, 323 (68,43\%) eram de $P$. juliflora.

Em relação à distribuição do número de indivíduos por classe de diâmetro para o componente adulto verificou-se predomínio de indivíduos na classe de 3 a $12 \mathrm{~cm}$ de diâmetro para a duas situações estudadas (Fig. 3A). Dos 441 indivíduos registrados no Ambiente II, $388(87,98 \%)$ pertenciam a $P$. juliflora. Quanto à regeneração natural, constata-se que a maior concentração de indivíduos ocorreu na classe de

A
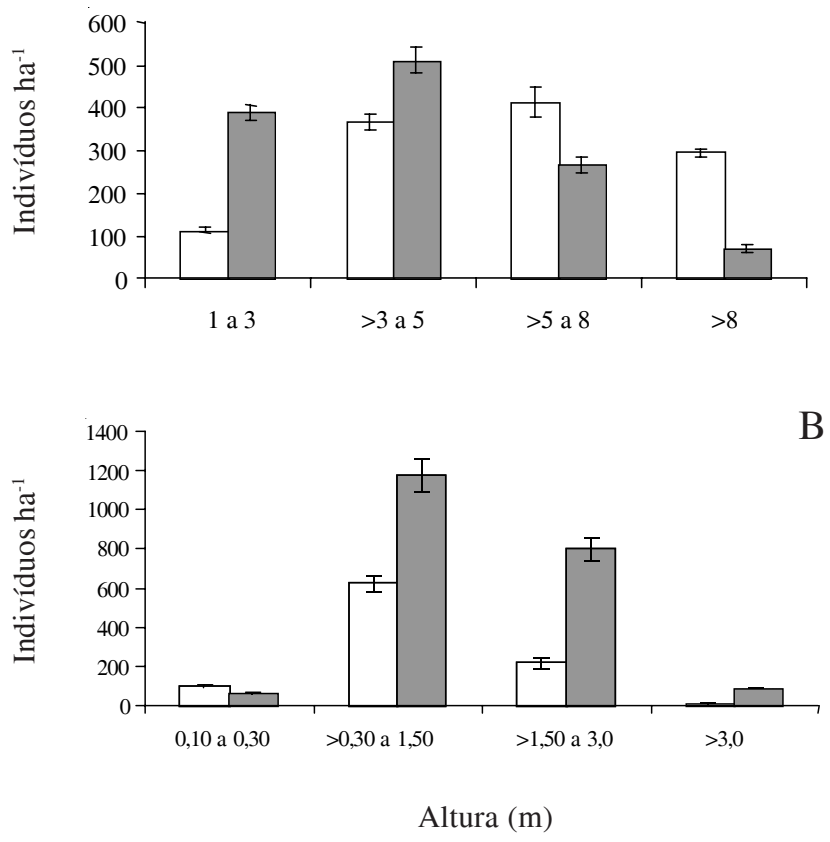

Figura 2. Distribuição do número de indivíduos por hectare, por classes de altura, em dois Ambientes de caatinga no Município de Monteiro, PB. Ambiente I: caatinga sem a presença de $P$. juliflora (Sw.) DC.; Ambiente II: caatinga invadida por P. juliflora. $\mathrm{A}=$ Adultos e $\mathrm{B}=$ Regeneração Natural. Os segmentos verticais sobre as barras representam o desvio padrão. ( $\square=$ Ambiente I; = Ambiente II). 
diâmetro de 0,30 a 1,50 cm, o que corresponde também às menores classes de tamanho (Fig. 3B), em ambas as Áreas. Neste componente, foi constatada, igualmente, uma grande proporção de indivíduos de $P$. juliflora, pois dos 444 indivíduos registrados, 325 $(73,19 \%)$ pertenciam àquela espécie.

Segundo Faria et al. (2001), regeneração é um processo que inclui não só a sucessão secundária, mas também formas de manipulação florestal que promovam novos e maiores estádios produtivos do crescimento da mata. Para que ocorra a regeneração natural em uma área degradada ou em processo de degradação, são necessárias algumas condições, como o cessamento dos processos causadores da degradação (pastoreio e incêndio), existência de fontes de propágulos (banco de sementes, chuva de sementes) e dispersores, boas condições microclimáticas e edáficas, ausência de predadores para o estabelecimento e ocorrência do ciclo de vida completo das plântulas. No caso específico da invasão da caatinga por $P$. juliflora, observações dos autores e comunicações de pessoas residentes nas regiões afetadas indicam que a invasora não consegue ocupar a caatinga conservada.
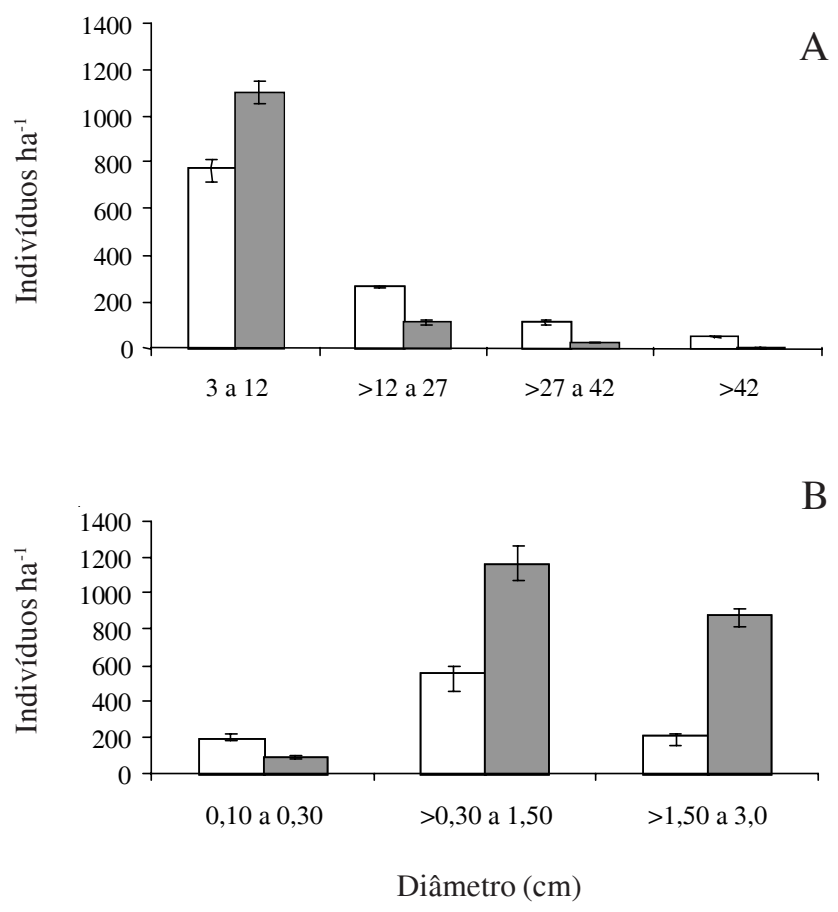

Figura 3. Distribuição do número de indivíduos por hectare, por classes de diâmetro, em dois Ambientes de caatinga no Município de Monteiro, PB. Ambiente I: caatinga sem a presença de $P$. juliflora (Sw.) DC.; Ambiente II: caatinga invadida por $P$. juliflora. $\mathrm{A}=$ Adultos e $\mathrm{B}=$ Regeneração Natural. Os segmentos verticais sobre as barras representam o desvio padrão. ( $\square=$ Ambiente I; = Ambiente II).

\section{Agradecimentos}

Os autores agradecem à Fundação $\mathrm{O}$ Boticário de Proteção à Natureza, pelo apoio financeiro ao projeto (processo 0582/20031) que possibilitou a execução deste trabalho; ao CCA/UFPB; ao Senhor Jorge Menezes e à família Lafayete, proprietários das áreas estudadas.

\section{Referências bibliográficas}

Alcoforado Filho, F.G.; Sampaio, E.V.S.B. \& Rodal, M.J.N. 2003. Florística e fitossociologia de um remanescente de vegetação caducifólia espinhosa arbórea em Caruaru, Pernambuco. Acta Botanica Brasilica 17(2): 287-303.

Andrade, L.A.; Reis, M.G.F.; Reis, G.G. \& Souza, A.L. 1999. Classificação ecológica do Estado da Paraíba. 2 Delimitação e caracterização de sub - regiões ecológicas a partir de variáveis climáticas. Revista Árvore 23(2): 139-149.

Araújo, E.L.; Sampaio, E.V.S.B. \& Rodal, M.J.N. 1995. Composição florística e fitossociológica de três áreas de caatinga de Pernambuco. Revista Brasileira de Biologia 55(4): 595-607.

Azevedo, G.F. 1982. Como e porque a algarobeira foi introduzida no Nordeste. Pp. 300-306. In: F.C.E. Fonseca; A.P.B. Mota; C.F. Azêvedo; I. Trindade; J.F. Cruz \& R.A.L. Brito (eds.). Simpósio Brasileiro sobre Algaroba 1, 1982, Natal. Algaroba. Natal, EMPARN, (Empresa Agropecuária do Rio Grande do Norte. Documentos, 7).

Brasil, Ministério da Agricultura. 1971. Equipe de Pedologia e fertilidade do solo. Divisão de Agrologia - SUDENE. Levantamento exploratório: reconhecimento de solos do Estado da Paraíba. Rio de Janeiro, Boletim técnico, n. 15.

Brasil, Comissão Interministerial para Preparação da Conferência das Nações Unidas sobre Meio Ambiente e Desenvolvimento. 1991. O desafio do desenvolvimento sustentável: relatório do Brasil para a conferência das nações unidas sobre meio ambiente e desenvolvimento. Brasília, Secretaria de Imprensa Presidência da República.

Brasil, Ministério do Meio Ambiente. 2002. Avaliação e ações prioritárias para a conservação da biodiversidade da caatinga. Brasília, Secretaria de Biodiversidade e Florestas.

Brower, J.E. \& Zar, J.H. 1984. Field and laboratory methods for general ecology. Dubuque, W.M.C. Brow.

Cronk, Q.C.B. \& Fuller, J.L. 1995. Plant invaders. London, Chaman \& Hall.

Curtis, J.T. \& Macintosh, R.P. 1951. The interrelation of analytic and synthetic phytosociological characters. Ecology 31: 345-355.

Drumond, M.A.; Kiill, L.H.P. \& Nascimento, C.E.S. 2002. Inventário e sociabilidade de espécies arbóreas e arbustivas da Caatinga na Região de Petrolina, PE. Brasil Florestal 74: 37-43. 
Faria, H.H.; Sérgio, F.C. \& Garrido, M.A. 2001. Recomposição da vegetação ciliar integrada à conservação de microbacia. Revista do Instituto Florestal 21: 1-22.

Ferraz, E.M.N.; Rodal, M.J.N.; Sampaio, E.V.S.B. \& Pereira, R.C.A. 1998. Composição florística em trechos de vegetação de caatinga e brejo de altitude na região do Vale do Pajeú, Pernambuco. Revista Brasileira de Biologia 21(1): 7-15.

Finol, H. 1971. Nuevos parametros a considerarse en el analisis structural de las selvas virgenes tropicales. Revista Florestal Venezoelana 14(21): 29-42.

Gomes, P. 1961. A algarobeira. Rio de Janeiro, Ministério da Agricultura, Serviço de Informação Agrícola. (Serie SIA, 865)

Kent, M. \& Coker, P. 1999. Vegetation Description and Analysis - a pratical approach. Chichester, John Wiley \& Sons.

Krebs, C.J. 1989. Ecological methodology. New York, Harper and Row.

Lamprecht, H. 1962. Ensaio sobre unos métodos para el análisis estructural de los bosques tropicales. Acta Científica Venezuelana 13(2): 57-65.

Lamprecht, H. 1964. Ensayo sobre la estrutura florística de la parte Sur-Oriental del bosque universitario: El Caimital, Estado Barinas. Revista Florestal Venezuelana 7(10/11): 77-119.

Mateucci, S.D. \& Colma, A. 1982. Metodologia para el estudio de la vegetacion. Washington, Secretaria General de la Organizacion de los Estados Americanos. Programa Regional de Desarrolo Científico y Tecnológico.

Mccune, B. \& Mefford, M.J. 1999. PC-ORD version 4.0, multivariate analysis of ecological data, Users guide. MjM Software Design. Oregon, Glaneden Beach.

Muller-Dombois, D. \& Ellemberg, H. 1974. Aims and methods of vegetation ecology. New York, John Wiley \& Sons.

Noble, I.R. 1989. Attributes of invaders and the invading process: terrestrial and vascular plants. Pp. 301-313. In: J.A. Drake; H.A. Mooney; F. di Castri; R.H. Groves; F.J. Kruger; M. Rejmanek \& M.H. Williamson (eds.). Biological invasions: a global perspective. Wiley, Chichester.

Parker, I.M.; Simberloff, D.; Lonsdale, W.M.; Goodell, K.; Wonham, M.; Kareiva, P.M.; Williamson, M.H.; Vonholle, B.; Moyle, P.B.; Byres, J.E. \& Goldwasser, L. 1999. Impact: toward a framework for understanging the ecological effects of invaders. Biological Invasions 1: 3-19.

Pereira, I.M.; Andrade, L.A.; Barbosa, M.R.V. \& Sampaio, E.V.S.B. 2002. Composição florística e análise fitossociológica do componente arbustivo-arbóreo de um remanescente florestal no agreste paraibano. Acta Botanica Brasilica 16(3): 357-369.

Pivello, V.R.; Carvalho, V.M.N.C.; Peccinini, A.A.; Lopes, P.F. \& Rosso, S. 1999. Abundance and distribution of native and invasive alien grasses in a "cerrado" (Brazilian savanna) biological reserve. Biotropica 31(1): 71-81.
Randall, J.M. 1996. Weed control for the preservation of biological diversity. Weed technology 10: 370-383.

Rejmanek, M. \& Richardson, D.M. 1996. What attributes make some plant species more invasive? Ecology 77(6): $1655-1661$.

Rodal, M.J.N.F.; Sampaio, E.V.S.B. \& Figueiredo, M.A. 1992. Manual sobre métodos de estudos florísticos e fitossociológicos - ecossistema caatinga. Brasília, Sociedade Botânica do Brasil.

Rodal, M.J.N.; Andrade, K.V.A.; Sales, M.F. \& Gomes, A.P.S. 1998. Fitossociologia do componente lenhoso de um refúgio vegetacional no município de Buíque, Pernambuco. Revista Brasileira de Biologia 58(3): 517-526.

Roy, J. 1990. In search of the characteristics of plant invaders. Pp. 335-352. In: F. di Castri; A.J. Hansen \& M. Debussche (eds.). Biological invasions in Europe and the Mediterranean Basin. Dordrecht, Kluwer Academic Publishers.

Sampaio, E.V.S.B.; Sampaio, Y.; Vital, T.; Araújo, M.S.B. \& Sampaio, G.R. 2003. Desertificação no Brasil: conceitos, núcleos e tecnologias de recuperação e convivência. Recife, Ed. Universitária da UFPE.

Scolforo, J.R.S. \& Figueiredo Filho, A. 1998. Determinação e estimativa da área basal. Pp. 105-160. In: J.R.S. Scolforo \& A. Figueiredo Filho (eds.). Biometria florestal: medição e volumétrica de árvores. Lavras, Universidade Federal de Lavras / Fundação de Apoio ao Ensino, Pesquisa e Extensão.

Shiferaw, H.; Teketay, D.; Nemomissa, S. \& Assefa, F. 2004. Some biological characteristics that Foster the invasion of Prosopis juliflora (Sw.) DC. at Middle Awash Rift Valley Area, north-eastern Ethiopia. Journal of Arid Environments 58: 135-154.

Silva, S. 1997. Algarobeira. Natal, Serviço de Apoio ás Micro e Pequenas Empresas/Rio Grande do Norte.

Westbrooks, R. 1998. Invasive plants: changing the landscape of America: fact book. Washington, DC., Federal Interagency Committee for the Management of Noxious and Exotic Weeds.

Williamson, M. 1996. Biological invasions. London, Chapman \& Hall.

Williamson, M. \& Fitter, A. 1996. The characters of successful invaders. Biological Conservation 78: 163-170.

Zar, J.H. 1996. Biostatistical analysis. 3. ed. New Jersey, Prentice Hall.

Ziller, S.R. \& Galvão, F. 2002. A degradação da Estepe Gramíneo-Lenhosa no Paraná por contaminação biológica de Pinnus elliottii e P. taeda. Floresta 32(1): 41-47. 